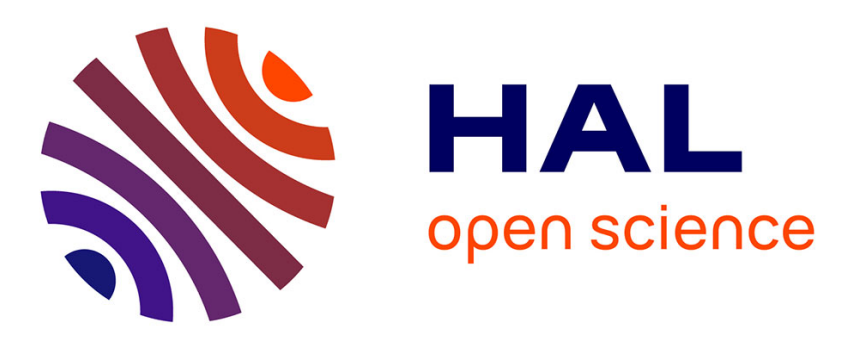

\title{
Signatures of Small Morphological Anisotropies in the Plasmonic and Vibrational Responses of Individual Nano-objects
}

\author{
Fabio Medeghini, Romain Rouxel, Aurélien Crut, Paolo Maioli, Francesco \\ Rossella, Francesco Banfi, Fabrice Vallée, Natalia del Fatti
}

\section{To cite this version:}

Fabio Medeghini, Romain Rouxel, Aurélien Crut, Paolo Maioli, Francesco Rossella, et al.. Signatures of Small Morphological Anisotropies in the Plasmonic and Vibrational Responses of Individual Nano-objects. Journal of Physical Chemistry Letters, 2019, 10 (18), pp.5372-5380. 10.1021/acs.jpclett.9b01898 . hal-02350956

\section{HAL Id: hal-02350956 https://hal.science/hal-02350956}

Submitted on 3 Nov 2020

HAL is a multi-disciplinary open access archive for the deposit and dissemination of scientific research documents, whether they are published or not. The documents may come from teaching and research institutions in France or abroad, or from public or private research centers.
L'archive ouverte pluridisciplinaire HAL, est destinée au dépôt et à la diffusion de documents scientifiques de niveau recherche, publiés ou non, émanant des établissements d'enseignement et de recherche français ou étrangers, des laboratoires publics ou privés. 


\title{
Signatures of Small Morphological Anisotropies \\ in the Plasmonic and Vibrational Responses of Individual Nano-Objects
}

\author{
Fabio Medeghini ${ }^{1+}$, Romain Rouxel ${ }^{1}$, Aurélien Crut ${ }^{1^{*}}$, Paolo Maioli ${ }^{1}$, Francesco Rossella ${ }^{2}$, Francesco \\ Banfi $^{1,3}$, Fabrice Vallée ${ }^{1}$ and Natalia Del Fatti ${ }^{1}$ \\ ${ }^{1}$ FemtoNanoOptics group, Université de Lyon, CNRS, Université Claude Bernard Lyon 1, Institut Lumière \\ Matière, F-69622 Villeurbanne, France \\ ${ }^{2}$ NEST, Scuola Normale Superiore and Istituto Nanoscienze-CNR, Piazza S. Silvestro 12, I-56124 Pisa, \\ Italy \\ ${ }^{3}$ Interdisciplinary Laboratories for Advanced Materials Physics (I-LAMP), Università Cattolica del Sacro \\ Cuore, Brescia I-25121, Italy
}

\begin{abstract}
The plasmonic and vibrational properties of single gold nanodisks patterned on a sapphire substrate are investigated via spatial modulation and pump-probe optical spectroscopies. The features of the measured extinction spectra and time-resolved signals are highly sensitive to minute deviations of the nanodisk morphology from a perfectly cylindrical one. An elliptical nanodisk section, as compared to a circular one, lifts the degeneracy of the two nanodisk in-plane dipolar surface plasmon resonances, which can be selectively excited by controlling the polarization of the incident light. This splitting effect, whose amplitude increases with nanodisk ellipticity, correlates with the detection of additional vibrational modes in the context of time-resolved spectroscopy. Analysis of the measurements is performed through the combination of optical and acoustic numerical models. This allows us first to estimate the dimensions of the investigated nanodisks from their plasmonic response, and then to compare the measured and computed frequencies of their detectable vibrational modes, which are found in excellent agreement. This study demonstrates that single-particle optical spectroscopies are able to provide access to fine morphological characteristics, representing in this case a valuable alternative to traditional techniques aimed at post-fabrication inspection of subwavelength nanodevice morphology.
\end{abstract}

KEYWORDS: Nano-objects, single-particle spectroscopy, plasmonics, acoustic vibrations, anisotropy, nanodisks 
Nano-objects display unique physical properties (e.g. optical, vibrational, electronic, magnetic and thermal ones) that are intimately related to their composition, structure, morphology and environment. ${ }^{1-8}$ Unveiling the correlation between the morphological and physical properties of nanoobjects would on the one hand boost the engineering of nano-objects with tailored properties and on the other hand promote metrology protocols for their characterization, alternative to high-resolution imaging techniques such as electron and atomic force microscopies. For instance, measuring the period of the breathing vibrational modes of spherical nanoparticles using time-resolved spectroscopy allows the precise determination of their diameter, exploiting the proportionality between these two quantities predicted by continuum mechanics and experimentally shown to be preserved down to 1 $\mathrm{nm}$ nanoparticle sizes. ${ }^{8-13}$ Ultrafast optical methods have recently emerged as competitive techniques for devices nanometrological inspection. ${ }^{14,15}$ Nevertheless, the focus has mainly been on retrieving the thermal and mechanical properties of metallic nanometric sized films, ${ }^{16-18}$ overlayers ${ }^{19}$ and periodic nanostructures. ${ }^{20,21}$

In this context, the development of optical spectroscopy approaches capable to investigate the plasmonic and vibrational responses of individual metal nano-objects has been a major breakthrough, as they avoid spurious averaging and inhomogeneous broadening effects resulting from dispersions of nano-object morphology, environment and orientation in ensemble of nanostructures. Several techniques have been developed to measure the scattering, absorption and extinction (sum of the two former processes) spectra of single nano-objects, as for instance dark-field, photothermal and spatial modulation spectroscopies. ${ }^{22-29}$ Investigation of the vibrational modes of single nano-objects has been demonstrated using time-resolved spectroscopy $\mathrm{y}^{8,30-33}$ and, more recently, inelastic light scattering. ${ }^{34,35}$ Noticeably, the use of such single-particle approaches allows to shed light onto processes which can hardly be investigated in ensemble measurements, such as the polarization dependence of the optical properties of nano-objects, sensitive to their shape and orientation ${ }^{36-39}$ and the quality factors of their plasmonic and vibrational modes. ${ }^{8,40-46}$ Quantitative polarization-resolved extinction measurements using spatial modulation spectroscopy enable optical characterization of the dimensions and orientation of simple nano-objects (nanorods and nanospheres) placed in a known environment and, vice versa, to measure the refractive index of the local environment if the morphology of the nanoobject has been previously determined. ${ }^{29,39-41,47}$

The present work exploits the assets of single-particle studies to address the impact that slight deviations of a nano-object morphology from an ideal one (here, a perfect cylinder) have on its plasmonic and vibrational responses. The technological drive underlying this research stands in the quest for a fast and non-destructive nanometrology tool for nano-object morphology post-fabrication inspection. The morphology of sub-100 nm size nanostructures defined by electron beam lithography 
(EBL) - such as those considered here - often displays deviations from the targeted shape, due to the standard $\approx 5-10 \mathrm{~nm}$ resolution limit achievable in practice with this technique. ${ }^{48}$ Additionally, systematic errors may also affect the positioning, size and intensity of the electron beam scanned across the sample surface.$^{49}$ Characterization of the fabricated nano-objects is routinely performed by scanning/transmission electron or atomic force microscopies (SEM/TEM or AFM). However, both these techniques have limitations. Specifically, although AFM grants an excellent vertical resolution, it displays two main drawbacks: a relatively poor lateral resolution and high acquisition times, due to tip convolution effects and scanning. TEM yields very high resolution but can only be applied to thin samples. This limitation does not apply to SEM imaging, whose resolution is however lower than the TEM one and severely affected by sample charging issues when SEM is applied to nano-objects on insulating substrates. Developing fast and sensitive optics-based methods capable of detecting deviations from sought shapes of nano-objects supported on thick and/or dielectric substrates constitutes therefore a relevant technological challenge. Here, we show that far-field optical methods are especially relevant to the case of highly symmetric nano-objects such as cylinders, which present a rotational invariance about their axis. In particular, we demonstrate that a minute deviation of the nano-object section from a circular one has clear signatures in both its plasmonic and vibrational responses.

The present study combines scanning electron microscopy (SEM) imaging and linear and timeresolved optical experiments on individual gold nanodisks (NDs) lithographically defined by EBL on a thick (480 $\mu \mathrm{m}$ thickness) sapphire substrate. Optical investigations were performed using experimental methods similar to those used in our recent study of the vibrational quality factors of circular NDs. ${ }^{44}$ Namely, the extinction spectra of individual NDs were quantitatively measured using spatial modulation spectroscopy (SMS), ${ }^{24,28}$ a far-field optical technique involving the modulation of the position of a single nanoparticle placed in the focal plane of a tightly focused laser beam (Fig. 1a). Additionally, the ND ultrafast dynamics was monitored using single-particle femtosecond pump-probe spectroscopy. ${ }^{50}$ This approach is based on the illumination of the investigated nanoparticle with two spatially overlapping, tightly focused and time-delayed femtosecond light pulses, referred to as pump and probe (Fig. 1b). It provides access to the frequencies and quality factors of the detected ND vibrational modes $32,33,42,46$. These optical measurements were performed on a set of individual NDs with the same nominal dimensions $(D \approx 70 \mathrm{~nm}$ diameter and $\mathrm{h} \approx 20 \mathrm{~nm}$ thickness, these dimensions being chosen because circular NDs of this size present a simple ultrafast response dominated by a single vibrational mode ${ }^{44}$ ), nanopatterned with a single fabrication session including EBL, Au thermal evaporation and lift-off. The fabrication process yields to unavoidable dispersion of ND size and morphology at the nanometer scale; optical investigations allow us to demonstrate that minute 
deviations from a circular ND cross-section have marked and correlated signatures in the plasmonic and vibrational responses of the nano-object.

Combined SEM and optical spectroscopy investigations were performed on seven NDs. Fig. 2 reports the results obtained for the two investigated NDs displaying (in view of their optical response, see below) minimal and maximal deviations from a perfectly circular shape, referred to as ND A and ND B, respectively. The SEM images of these two NDs are shown in Fig. 2a,c. The two images were acquired by setting machine parameters typically yielding a lateral resolution of few nanometers. However, due to the insulating character of the sapphire substrate, the electrons from the scanned beam are prevented from leaving the ND, instead accumulating on its surface. This charging of the sample eventually induces spurious effects in the generation of the secondary electrons, whose detection is at the core of SEM image generation. This process dramatically degrades lateral resolution, which can exceed $15 \mathrm{~nm}$, thus preventing precise characterization of the section of a nominally $\approx 70$ nm wide ND. In particular, no clear deviation from a circular shape can be detected on the SEM micrographs shown in Fig. 2a,c, radial profiles extracted from them (Fig. 2b,d) showing similar widths $(\approx 70 \mathrm{~nm} F W H M)$ in all cases.

The dependence of the extinction cross-section $\sigma_{\text {ext }}$ of the NDs on light polarization and wavelength, measured using SMS, is presented in Fig. 2e-h. Investigations of $\sigma_{\text {ext }}$ polarization dependence were performed by measuring its value as a function of the angle $\theta$ between a fixed direction in the plane of the sample and incident light polarization direction at a $\lambda=700 \mathrm{~nm}$ wavelength (polar plots in Fig. 2e,g). The polarization-independent extinction observed for ND A (Fig. 2e) is consistent with a circular section for this ND. In contrast, ND B extinction shows sinusoidal variations of large amplitude with $\theta$, resulting in a highly elongated polar plot in Fig. $2 \mathrm{~g}$. This result clearly indicates that ND B does not have a perfectly circular section.

Fig. $2 \mathrm{f}$,h shows the extinction spectra of ND A and ND B, measured for the two polarization directions yielding minimal/maximal extinction at $\lambda=700 \mathrm{~nm}$. All spectra present resonances with Lorentzian shapes when plotted in the energy domain (Lorentzian fits are shown as lines in Fig.2f,h), ascribed to the two in-plane dipolar surface plasmon resonances (SPRs) of the individual NDs. The extinction spectrum of ND A is found to be approximately the same for both polarizations considered (with central SPR positions $\lambda_{1} \approx \lambda_{2} \approx 640 \mathrm{~nm}$ ). The polarization independence of ND A optical response over a large spectral range confirms its circular section (note that this observation is not in itself an absolute proof of the circular character of ND A section, as non-circular sections characterized by discrete rotational symmetries, such as nanotriangles and nanocubes, may also yield a polarizationindependent response; ${ }^{51-53}$ however, such morphologies are not expected to arise from the fabrication 
process). In contrast, the two resonances measured for ND B are spectrally shifted, with central positions $\lambda_{1} \approx 660 \mathrm{~nm}$ and $\lambda_{2} \approx 690 \mathrm{~nm}$, corresponding to a relative shift $\left(\lambda_{2}-\lambda_{1}\right) / \lambda_{1} \approx 5 \%$.

The vibrational dynamics of NDs A and B measured by pump-probe spectroscopy are shown in Fig. 2i-I. In these panels, the oscillating parts of the bare time-resolved signals, obtained upon subtraction of ND internal thermalization and cooling contributions, ${ }^{44}$ are plotted (black dotted lines). For ND A, the deduced oscillating signal is well fitted by a single damped sinusoid of frequency $f_{1}=23.3$ $\mathrm{GHz}$ and quality factor $\mathrm{Q}_{1}=14$ (with quality factor $\mathrm{Q}$ defined as $\mathrm{Q}=\pi \mathrm{f} \tau$, where $\tau$ is the damping time constant). Conversely, the signal obtained for ND B displays a marked beating pattern, which can be reproduced by summing two damped sinusoids of close frequencies $\left(f_{1}=19.2 \mathrm{GHz}\right.$ and $f_{2}=22.6 \mathrm{GHz}$ ) and quality factors $\left(Q_{1}=21\right.$ and $\left.Q_{2}=16\right)$. These findings are confirmed by Fourier analysis of the measured signals, showing one and two peaks for ND A and ND B, respectively (Fig.2j,I).

The observations summarized in Fig. 2 demonstrate that the morphological, plasmonic and vibrational properties of NDs are highly correlated. Indeed, circular NDs (such as ND A) display two degenerate dipolar SPRs and lead to the detection of a single vibrational mode, while deviation from a circular section (as in ND B case) lifts the SPR resonance degeneracy and leads to the detection of a supplementary vibrational mode. Summaries of plasmonic and vibrational quantities measured for the entire set of investigated individual NDs are shown in Fig. 3a (black squares) and Fig. $4 \mathrm{f}$ (orange squares and green circles), respectively. Fig. 3a provides an overview of their SPR central wavelengths $\lambda_{1}$ and $\lambda_{2}$. They span the $630-700 \mathrm{~nm}$ range, while relative resonance shifts $\left(\lambda_{2}-\lambda_{1}\right) / \lambda_{1}$ range between $<0.5 \%$ (for ND A) and 5\% (for ND B). The extinction spectrum of a third ND (labeled ND C) for which $\left(\lambda_{2}-\lambda_{1}\right) / \lambda_{1}$ $=1.4 \%$ (i.e., much smaller than in ND B case) is shown in Fig. 3b. Interestingly, maximal extinction at $700 \mathrm{~nm}$ wavelength was obtained for $\theta \approx 60^{\circ}$ light polarization angle (as in ND B case, Fig. $2 \mathrm{j}$ and $3 \mathrm{~b}$ ) for all investigated NDs. This allows one to ascribe ND elliptical cross-sections to a systematic fabrication effect, likely a drift of parameters of the electron beam (e.g. astigmatism) during exposure, leading to a slight elongation of the NDs along a constant direction. The monomodal or bimodal character of the oscillating part of time-resolved signals well correlates with the relative SPR shifts $\left(\lambda_{2}-\lambda_{1}\right) / \lambda_{1}$. Indeed, a single vibrational mode with $\approx 20 \mathrm{GHz}$ frequency was detected for the two NDs with the lowest relative shifts, i.e. with almost circular sections (NDs $A$ and $C$ ), while a beating pattern, arising from comparable responses of two distinct modes, was detected for all other NDs (Fig. 4f).

The detailed analysis of the experiments illustrated in Fig. 2 was accomplished in two steps. First, the extinction spectra measured on each investigated ND were compared to the results of finiteelement modeling (FEM) optical calculations, in order to estimate the ND size and ellipticity. In a second step, an acoustic FEM model was used to compute the vibrational spectra of NDs in the context 
of a pump-probe experiment. This clarified the effect of an elliptical section and allowed a direct comparison of the measured vibrational frequencies with simulated ones, using ND dimensions deduced from the initial optical analyses.

FEM simulations of ND plasmonic response (using the RF module of the COMSOL Multiphysics commercial software) were performed by modeling gold NDs as elliptic cylinders characterized by three size parameters: their height $h$, and the minor and major axis (diameters) of their elliptical crosssection $D_{1}$ and $D_{2}$. Their inhomogeneous environment resulting from the fact that they are supported on a sapphire substrate was explicitly included in the model, refractive indexes of 1.76 and 1 being used for the sapphire substrate and the above medium, respectively. Note that using the refractive index of air for the above local medium is expected to be reliable here only because lithographed objects are considered. In contrast, using a higher value (which can reach 1.3) was necessary to reproduce the extinction spectra of chemically synthesized nano-objects, presumably because of the presence of surfactants and/or residual solvent around them $\left.{ }^{29,39,47}\right)$. Gold dielectric function $\varepsilon$ was modeled based on Johnson and Christy tables of bulk gold dielectric function $\varepsilon^{\mathrm{JC}}{ }^{54}$ Specifically, the expression $\varepsilon(\omega)=\varepsilon^{\lrcorner c}(\omega)+\omega_{p}{ }^{2} /\left(\omega\left(\omega+i \gamma_{0}\right)\right)-\omega_{p}{ }^{2} /(\omega(\omega+i \gamma))$ was used, which corresponds to describing the dielectric response of gold conduction electrons by a Drude term ${ }^{29}$ involving the bulk gold plasma frequency $\omega_{P}\left(\hbar \omega_{P}=9.01 \mathrm{eV}\right)$ and an optical scattering rate $\gamma$ of the conduction electrons larger than that of bulk gold ( $\left.\hbar \gamma_{0} \approx 50 \mathrm{meV}\right) .{ }^{41}$ We found that using $\hbar \gamma \approx \hbar \gamma_{0}+100$ meV was necessary to reproduce experimental SPR widths. This increase of $\hbar \gamma$ as compared to its bulk value cannot be ascribed exclusively to quantum confinement effects, which are expected to only induce $\approx 20$ meV broadening for the ND sizes used. ${ }^{40,41,55}$ It likely originates from crystalline defects in the investigated nano-objects (the fabricated metal NDs being disordered agglomerates of crystal grains rather than perfect single crystals due to the thermal evaporation process), which are known to broaden SPRs. ${ }^{56}$ ND illumination by a linearly polarized plane wave was considered. Calculations were performed for incident light polarization aligned along the major and minor axis of the ND elliptical section. Absorption and scattering cross-sections were deduced from the computed electric field and summed to yield the extinction one.

FEM optical simulations were first performed for NDs with various $\mathrm{h}(20 \mathrm{~nm}$, full circles in Fig. $3 a$ and $25 \mathrm{~nm}$, hollow circles), $D_{1}(70,75,80,85$ and $90 \mathrm{~nm})$ and $D_{2} / D_{1}(1,1.05,1.1$ and 1.15$)$ values (with $\hbar \gamma=\hbar \gamma_{0}+100 \mathrm{meV}$ ). These simulations yield SPRs with $\lambda_{1}$ and $\lambda_{2}$ positions in the $600-730 \mathrm{~nm}$ range, encompassing the 630-700 nm experimental one (Fig. 3a). This analysis shows that in the investigated size range $\lambda_{1}$ is mainly determined by $D_{1} / h$, while the $\lambda_{2} / \lambda_{1}$ ratio is primarily determined by $D_{2} / D_{1}$, regardless of the $h$ value (as demonstrated by the alignment of the SPR positions computed for a given $D_{2} / D_{1}$ value in Fig. 3a). Comparison with the experimental results thus suggests that $D_{2} / D_{1}$ ranges 
between 1 and 1.15 for the investigated NDs. Further optical simulations of ND extinction crosssections were performed with the scope of analyzing the quantitative spectra measured on each investigated ND (Fig. 3b). The ND size parameters ( $h, D_{1}$ and $\left.D_{2}\right)$ used in the modeling were tuned so as to optimally reproduce the positions and areas of experimental resonances obtained for each ND. The outcome is illustrated in Fig. 3b for the cases of two specific NDs (ND B and ND C), showing the good match between the experimental extinction spectra and the simulated ones. The values determined for $h$ and $D_{1}\left(h=21.3 \mathrm{~nm}, D_{1}=79.9 \mathrm{~nm}\right.$ for ND $B, h=23.7 \mathrm{~nm}, D_{1}=78.3 \mathrm{~nm}$ for ND C) are close for the two NDs, the $D_{1}$ value being about $10 \%$ larger than the nominal $70 \mathrm{~nm}$ one. $D_{2} / D_{1}$ values of 1.04 and 1.14 are found for ND C and ND B, respectively. In view of this analysis, the combination of SMS with numerical modeling appears as a powerful tool for detecting and quantifying the elongation of a subwavelength nano-object. Moreover this approach is sensitive and able to detect minute ellipticities (e.g. a $4 \%$ difference between $D_{2}$ and $D_{1}$, corresponding to a detectable $1.5 \%$ shift between $\lambda_{1}$ and $\lambda_{2}$, as shown in Fig. $3 b$ for ND C).

Acoustic FEM simulations based on the Structural Mechanics module of COMSOL were performed by extending the model that we previously used for NDs with a circular cross-section ${ }^{44}$ to the case of elliptic cylinders. The key ingredients of the model remain the same, vibrational spectra being obtained by computing the average elastic energy stored in a ND as a function of the frequency of a periodical excitation corresponding to a homogeneously excited ND. A perfectly matched layer was used at the border of the simulation domain to avoid spurious acoustic reflections due to environment truncation. A perfect mechanical contact (i.e., continuity of displacement and normal stress) was assumed at the ND/substrate interface, and stress-free boundary conditions were used on all other ND faces. However, in contrast to our previous study, ${ }^{44}$ the simulated system lacks axial symmetry when elliptic cylinders are considered, so that a 3D model has to be used instead of a 2D one, at the cost of a much higher number of mesh elements (about $10^{5}$ in the $3 \mathrm{D}$ model), and thus much longer computational times.

Fig. 4a-e presents the vibrational spectra obtained for $\mathrm{h}=20 \mathrm{~nm}$ NDs with increasing ellipticity $\left(D_{2} / D_{1}=1,1.02,1.05,1.1\right.$ and 1.15). Simulations were performed for constant ND volume and section area, maintaining the equivalent diameter $D_{\text {eq }}=\left(D_{1} D_{2}\right)^{1 / 2}$ fixed to $80 \mathrm{~nm}$ in all calculations. The vibrational spectrum computed in the circular ND case $\left(D_{2} / D_{1}=1\right.$, Fig. $\left.4 a\right)$ is dominated by a single vibrational mode with a frequency $f_{1}=21 \mathrm{GHz}$ and a quality factor $Q_{1}=17$, referred to as mode 1 in the following. It involves a radially isotropic displacement in sections parallel to the substrate surface (Fig. 4 ) inducing both ND deformation and motion of the ND/substrate interface. The large displacement in the substrate associated to mode 1 (Fig. 4) shows that it efficiently converts ND vibrations into acoustic waves in the substrate, explaining its moderate quality factor $Q_{1}$. As shown in Fig. $4 b$-e, increase of 
$D_{2} / D_{1}$ at fixed $D_{\text {eq }}$ does not significantly affect the main characteristics of mode 1 . However, $D_{2} / D_{1}$ increase has a clear effect on vibrational spectra, namely the progressive emergence of two additional modes (called modes 2 and 3 ) at frequencies slightly above $f_{1}\left(f_{2} \approx 23 \mathrm{GHz}\right.$ and $f_{3} \approx 24 \mathrm{GHz}$ for $\left.D_{2} / D_{1}=1.15\right)$, not excited in the circular ND case. The integrated energy spectral density of mode 2 is typically five times larger than that of the mode 3 , and reaches $65 \%$ of that of mode 1 for a ratio $D_{2} / D_{1}=1.15$. Its computed displacement profile is strongly anisotropic and mostly localized close to the top ND surface (Fig. 4e). Its predicted quality factor $\mathrm{Q}_{2}$ exceeds 1000 . Such a high value is consistent with the small associated displacement at the ND/substrate interface, considerably limiting acoustic emission in the substrate (the only damping mechanism considered in the FEM model). Similarly to the frequency $f_{1}$ of mode 1 , the frequency $f_{2}$ of mode 2 is weakly sensitive to the $D_{2} / D_{1}$ ratio at fixed $D_{\text {eq, }}$, decreasing by only $0.5 \%$ from $D_{2} / D_{1}=1.02$ to $D_{2} / D_{1}=1.15$. The fact that $f_{1}$ and $f_{2}$ are quasi-independent of $D_{2} / D_{1}$ means that neither the absolute values of these frequencies nor their relative shift can be exploited for a quantitative measurement of morphological anisotropies (at variance with plasmonic measurements, where the shift between in-plane dipolar SPRs is directly related to ND section ellipticity). Conversely, the relative weights of these modes in the vibrational excitation spectra are much more sensitive to anisotropy. These are however hard to extract from experimental signals, as mode amplitudes are affected by both excitation and detection processes in time-resolved experiments. . $^{8,14,57}$

These simulations clarify the detection of multiple modes with close frequencies for noncircular NDs. In view of their frequencies (close to $20 \mathrm{GHz}$ and differing by about $10 \%$ ), the two modes dominating the time-resolved signals measured for non-circular NDs are ascribed to modes 1 and 2, which present dominant amplitudes in simulated vibrational spectra (mode 3 does not clearly stem out in experiments, presumably because of its smaller integrated energy spectral density and the proximity of its frequency with that of mode 2). A thorough comparison between the experimental and computed frequencies of modes 1 and 2 requires to individually analyze each investigated ND (as each one has a slightly different morphology), and to separate the effect of ND ellipticity ( $D_{2} / D_{1}$ ratio) from that of ND size (absolute values of $h, D_{1}$ and $D_{2}$ ). To this end, the $f_{1}$ and $f_{2}$ vibrational frequencies measured for each ND were normalized against the frequency $f_{1, \text { circ }}$ computed for a circular ND of thickness $h$ and equivalent diameter $D_{e q}=\left(D_{1} D_{2}\right)^{1 / 2}$ (i.e. of same volume and section area) using the optically determined values of $h, D_{1}$ and $D_{2}$ (Fig. $4 f$ ). The same treatment was applied to FEM-computed frequencies (Fig. 4a-e), yielding $f_{1} / f_{1, \text { circ }} \approx 1$ and $f_{2} / f_{1, \text { circ }} \approx 1.1$ in the $D_{2} / D_{1}$ range of the experiments (squares and circles in Fig. 4f). A good agreement is observed between the experimental and simulated normalized frequencies. Such agreement is remarkable considering the multiphysics character of the analysis, which combines optical and vibrational models. In particular, the normalized experimental $f_{1}$ values are approximately independent of $D_{2} / D_{1}$, which is fully consistent with the FEM-predicted 
invariance of $f_{1}$ (for constant $D_{\text {eq }}$ ) illustrated by Fig. 4a-e. Moreover, the normalized experimental frequencies $f_{1} / f_{1, \text { circ }}$ always remain between 0.9 and 1.1 , which means that the complete opto-acoustic model used in the analysis allows for the reproduction of the measured $f_{1}$ values with maximal deviations of $10 \%$. These discrepancies roughly correspond to the estimated errors affecting reduced frequency determination, resulting from both ND size determination (from SMS measurements) and extraction of vibrational frequencies (from time-resolved experiments). Similar good agreement is obtained for $f_{2}$ values (note however that the computed value of $f_{2} / f_{1, \text { circ }}$ depends on the $D_{\text {eq }} / h$ ratio used in the simulations).

We finally briefly address the vibrational quality factors of non-circular NDs. In time-resolved experiments, the measured $Q$ values are limited by two distinct categories of damping processes: radiative damping (i.e., emission of acoustic waves in the nano-object environment, associated to a $Q_{\text {env }}$ quality factor) and intrinsic damping (associated to a quality factor $Q_{\text {int, }}$ whose dominant mechanisms still remain unclear). ${ }^{8,42,44,45}$ Assuming the two mechanisms to be independent leads to $1 / Q=1 / Q_{\text {env }}+1 / Q_{\text {int. }}$. Since no intrinsic damping is included in the FEM simulations, they only model $Q_{\text {env }}$ and cannot be directly compared with experimental $Q$ values, unless radiative damping is the dominant damping source (i.e. if $Q_{\text {int }}>>Q_{\text {env }}$ ). Here, the proximity between the quality factors measured for mode $1\left(Q_{1}=18 \pm 5\right)$ and the computed one (17) indicates that this is the case for this mode, a conclusion also supported by our previous estimation of $\mathrm{Q}_{\text {int }} \approx 70$ for mode 1 in the context of investigations limited to circular NDs. ${ }^{44}$ Conversely, the measurements yield $Q_{2}=16 \pm 3$ quality factors for mode 2 , considerably smaller than those obtained in simulations $\left(Q_{2}>1000\right.$ for $\left.D_{e q} / h=4\right)$. This could result from strong intrinsic damping, with an associated $Q_{\text {int }} \approx 16$ quality factor smaller than for mode 1 . However, this is not the only possible explanation for the observation of $Q_{2} \approx Q_{1} \approx 20$, as it is also likely that small deviations of the actual ND shape from the ideal one (elliptic cylinder) considered in the simulations would destroy the localized character of mode 2 displacement field (Fig. 4) and therefore make its $Q_{\text {env }}$ value smaller, and closer to that of mode 1 . A detailed investigation of the damping mechanisms of such acoustic vibrations remains an important challenge, but is out of the scope of this paper.

In conclusion, we have experimentally shown and numerically rationalized that the occurrence of small deviations from a high-symmetry morphology in a set of individual EBL-defined metal NDs of nominally circular section can be optically detected and quantified. Even a minute $(<5 \%)$ morphological ND ellipticity translates into both plasmonic and acoustic signatures, highlighting analogies between these two physical responses. Polarization-resolved single-particle extinction measurements show a splitting of the ND in-plane SPRs. This splitting augments with ND ellipticity, thus serving as an ideal tool to spot-out deviations from the sought circular geometry. Comparison of experimental spectra with those computed using FEM allows retrieving all the ND geometric parameters (i.e. $h, D_{1}$ and $D_{2}$ 
under the assumption of an elliptic cylinder shape). A correlated signature of ellipticity is the appearance of a beating oscillations in time-resolved pump-probe signals, associated to the excitation and detection of an additional vibrational mode as compared to the perfectly circular ND case. This allows to discriminate deviations from perfect circularity on the basis of ultrafast spectroscopy. In the latter case, the quantitative determination of ellipticity is however not trivial, as the frequency of the detected modes weakly depends on ND anisotropy at fixed $D_{\text {eq. }}$. Comparison with FEM modeling demonstrates that the measured frequencies are in good agreement with those predicted using ND sizes extracted from the analysis of extinction spectra. The methods here presented may readily be extended to other nano-object shapes, encompassing those presenting continuous or discrete rotational symmetries such as nanospheres, nanowires, nanotriangles, nanocubes, and thus with a polarization-independent optical response. ${ }^{51}$ Indeed, in this case deviations from an ideal morphology will make the optical response polarization-dependent, and increase the number of excitable vibrational modes, as in the investigated ND case.

\section{AUTHOR INFORMATION}

\section{Corresponding author}

*E-mail: aurelien.crut@univ-lyon1.fr

\section{ORCID}

Fabio Medeghini : 0000-0002-0508-3365

Aurélien Crut: 0000-0003-2185-709X

Paolo Maioli: 0000-0002-4199-8810

Francesco Rossella: 0000-0002-0601-4927

Francesco Banfi: 0000-0002-7465-8417

Natalia Del Fatti: 0000-0002-8074-256X

\section{Present Addresses}

† F. M.: JILA, University of Colorado Boulder and National Institute of Standard and Technology, Boulder, Colorado 80309, USA

\section{Notes}

The authors declare no competing financial interest.

\section{ACKNOWLEDGMENTS}


This work was supported by the LABEX iMUST (ANR-10-LABX-0064) of Universite de Lyon, within the program "Investissements d'Avenir" (ANR-11-IDEX-0007) operated by the French National Research Agency (ANR). F.B and F.R. acknowledge financial support from the MIUR Futuro in ricerca 2013 Grant in the frame of the ULTRANANO Project (Project No. RBFR13NEA4) and support from Universita Cattolica del Sacro Cuore through D.2.2 and D.3.1 grants.

\section{REFERENCES}

(1) Voisin, C.; Christofilos, D.; Del Fatti, N.; Vallée, F.; Prével, B.; Cottancin, E.; Lermé, J.; Pellarin, M.; Broyer, M. Size-Dependent Electron-Electron Interactions in Metal Nanoparticles. Phys. Rev. Lett. 2000, 85, 2200-2203.

(2) Kelly, K. L.; Coronado, E.; Zhao, L. L.; Schatz, G. C. The Optical Properties of Metal Nanoparticles: The Influence of Size, Shape, and Dielectric Environment. J. Phys. Chem. B 2003, 107, 668-677.

(3) Cahill, D. G.; Ford, W. K.; Goodson, K. E.; Mahan, G. D.; Majumdar, A.; Maris, H. J.; Merlin, R.; Phillpot, S. R. Nanoscale Thermal Transport. J. Appl. Phys. 2003, 93, 793-818.

(4) Park, T.-J.; Papaefthymiou, G. C.; Viescas, A. J.; Moodenbaugh, A. R.; Wong, S. S. Size-Dependent Magnetic Properties of Single-Crystalline Multiferroic BiFeO 3 Nanoparticles. Nano Lett. 2007, 7, 766-772.

(5) Huang, X.; Jain, P. K.; El-Sayed, I. H.; El-Sayed, M. A. Gold Nanoparticles: Interesting Optical Properties and Recent Applications in Cancer Diagnostics and Therapy. Nanomedicine 2007, 2, 681-693.

(6) Hartland, G. V. Optical Studies of Dynamics in Noble Metal Nanostructures. Chem. Rev. 2011, $111,3858-3887$.

(7) Cahill, D. G.; Braun, P. V.; Chen, G.; Clarke, D. R.; Fan, S.; Goodson, K. E.; Keblinski, P.; King, W. P.; Mahan, G. D.; Majumdar, A.; et al. Nanoscale Thermal Transport. II. 2003-2012. Appl. Phys. Rev. 2014, 1, 011305.

(8) Crut, A.; Maioli, P.; Del Fatti, N.; Vallée, F. Acoustic Vibrations of Metal Nano-Objects: TimeDomain Investigations. Phys. Rep. 2015, 549, 1-43.

(9) Del Fatti, N.; Voisin, C.; Chevy, F.; Vallée, F.; Flytzanis, C.; Vallée, F. Coherent Acoustic Mode Oscillation and Damping in Silver Nanoparticles. J. Chem. Phys. 1999, 110, 11484-11487.

(10) Hodak, J. H.; Henglein, A.; Hartland, G. V. Size Dependent Properties of Au Particles: Coherent 
Excitation and Dephasing of Acoustic Vibrational Modes. J. Chem. Phys. 1999, 111, 8613-8621.

(11) Juvé, V.; Crut, A.; Maioli, P.; Pellarin, M.; Broyer, M.; Del Fatti, N.; Vallée, F. Probing Elasticity at the Nanoscale: Terahertz Acoustic Vibration of Small Metal Nanoparticles. Nano Lett. 2010, 10, 1853-1858.

(12) Sauceda, H. E.; Mongin, D.; Maioli, P.; Crut, A.; Pellarin, M.; Del Fatti, N.; Vallée, F.; Garzón, I. L. Vibrational Properties of Metal Nanoparticles: Atomistic Simulation and Comparison with TimeResolved Investigation. J. Phys. Chem. C 2012, 116, 25147-25156.

(13) Maioli, P.; Stoll, T.; Sauceda, H. E.; Valencia, I.; Demessence, A.; Bertorelle, F.; Crut, A.; Vallée, F.; Garzón, I. L.; Cerullo, G.; et al. Mechanical Vibrations of Atomically Defined Metal Clusters: From Nano- to Molecular-Size Oscillators. Nano Lett. 2018, 18, 6842-6849.

(14) Kelf, T. A.; Tanaka, Y.; Matsuda, O.; Larsson, E. M.; Sutherland, D. S.; Wright, O. B. Ultrafast Vibrations of Gold Nanorings. Nano Lett. 2011, 11, 3893-3898.

(15) Brick, D.; Engemaier, V.; Guo, Y.; Grossmann, M.; Li, G.; Grimm, D.; Schmidt, O. G.; Schubert, M.; Gusev, V. E.; Hettich, M.; et al. Interface Adhesion and Structural Characterization of Rolledup GaAs//n $\ln _{0.2} \mathrm{Ga}_{0.8}$ As Multilayer Tubes by Coherent Phonon Spectroscopy. Sci. Rep. 2017, 7, 18.

(16) Nardi, D.; Travagliati, M.; Murnane, M. M.; Kapteyn, H. C.; Ferrini, G.; Giannetti, C.; Banfi, F. Impulsively Excited Surface Phononic Crystals: A Route Toward Novel Sensing Schemes. IEEE Sens. J. 2015, 15, 5142-5150.

(17) Hoogeboom-Pot, K. M.; Turgut, E.; Hernandez-Charpak, J. N.; Shaw, J. M.; Kapteyn, H. C.; Murnane, M. M.; Nardi, D. Nondestructive Measurement of the Evolution of Layer-Specific Mechanical Properties in Sub-10 nm Bilayer Films. Nano Lett. 2016, 16, 4773-4778.

(18) Abadeer, N. S.; Murphy, C. J. Recent Progress in Cancer Thermal Therapy Using Gold Nanoparticles. J. Phys. Chem. C 2016, 120, 4691-4716.

(19) Travagliati, M.; Nardi, D.; Giannetti, C.; Gusev, V.; Pingue, P.; Piazza, V.; Ferrini, G.; Banfi, F. Interface Nano-Confined Acoustic Waves in Polymeric Surface Phononic Crystals. Appl. Phys. Lett. 2015, 106, 021906.

(20) Giannetti, C.; Banfi, F.; Nardi, D.; Ferrini, G.; Parmigiani, F. Ultrafast Laser Pulses to Detect and Generate Fast Thermomechanical Transients in Matter. IEEE Photonics J. 2009, 1, 21-32.

(21) Frazer, T. D.; Knobloch, J. L.; Hoogeboom-Pot, K. M.; Nardi, D.; Chao, W.; Falcone, R. W.; 
Murnane, M. M.; Kapteyn, H. C.; Hernandez-Charpak, J. N. Engineering Nanoscale Thermal Transport: Size- and Spacing-Dependent Cooling of Nanostructures. Phys. Rev. Appl. 2019, 11, 1.

(22) Sönnichsen, C.; Franzl, T.; Wilk, T.; von Plessen, G.; Feldmann, J.; Wilson, O.; Mulvaney, P. Drastic Reduction of Plasmon Damping in Gold Nanorods. Phys. Rev. Lett. 2002, 88, 077402.

(23) Boyer, D.; Tamarat, P.; Maali, A.; Lounis, B.; Orrit, M. Photothermal Imaging of Nanometer-Sized Metal Particles among Scatterers. Science 2002, 297, 1160-1163.

(24) Arbouet, A.; Christofilos, D.; Del Fatti, N.; Vallée, F.; Huntzinger, J.; Arnaud, L.; Billaud, P.; Broyer, M. Direct Measurement of the Single-Metal-Cluster Optical Absorption. Phys. Rev. Lett. 2004, 93, 127401 .

(25) Berciaud, S.; Cognet, L.; Blab, G. A.; Lounis, B. Photothermal Heterodyne Imaging of Individual Nonfluorescent Nanoclusters and Nanocrystals. Phys. Rev. Lett. 2004, 93, 257402.

(26) Hu, M.; Novo, C.; Funston, A.; Wang, H.; Staleva, H.; Zou, S.; Mulvaney, P.; Xia, Y.; Hartland, G. V. Dark-Field Microscopy Studies of Single Metal Nanoparticles: Understanding the Factors That Influence the Linewidth of the Localized Surface Plasmon Resonance. J. Mater. Chem. 2008, 18, 1949-1960.

(27) Slaughter, L.; Chang, W. S.; Link, S. Characterizing Plasmons in Nanoparticles and Their Assemblies with Single Particle Spectroscopy. J. Phys. Chem. Lett. 2011, 2, 2015-2023.

(28) Christofilos, D.; Blancon, J.-C.; Arvanitidis, J.; San Miguel, A.; Ayari, A.; Del Fatti, N.; Vallée, F. Optical Imaging and Absolute Absorption Cross Section Measurement of Individual NanoObjects on Opaque Substrates: Single-Wall Carbon Nanotubes on Silicon. J. Phys. Chem. Lett. 2012, 3, 1176-1181.

(29) Crut, A.; Maioli, P.; Del Fatti, N.; Vallée, F. Optical Absorption and Scattering Spectroscopies of Single Nano-Objects. Chem. Soc. Rev. 2014, 43, 3921-3956.

(30) Van Dijk, M. A.; Lippitz, M.; Orrit, M. Detection of Acoustic Oscillations of Single Gold Nanospheres by Time-Resolved Interferometry. Phys. Rev. Lett. 2005, 95, 1-4.

(31) Zijlstra, P.; Tchebotareva, A. L.; Chon, J. W. M.; Gu, M.; Orrit, M. Acoustic Oscillations and Elastic Moduli of Single Gold Nanorods. Nano Lett. 2008, 8, 3493-3497.

(32) Staleva, H.; Hartland, G. V. Vibrational Dynamics of Silver Nanocubes and Nanowires Studied by Single-Particle Transient Absorption Spectroscopy. Adv. Funct. Mater. 2008, 18, 3809-3817. 
(33) Burgin, J.; Langot, P.; Del Fatti, N.; Vallée, F.; Huang, W.; El-Sayed, M. A. Time-Resolved Investigation of the Acoustic Vibration of a Single Gold Nanoprism Pair. J. Phys. Chem. C 2008, $112,11231-11235$.

(34) Girard, A.; Gehan, H.; Mermet, A.; Bonnet, C.; Lermé, J.; Berthelot, A.; Cottancin, E.; Crut, A.; Margueritat, J. Acoustic Mode Hybridization in a Single Dimer of Gold Nanoparticles. Nano Lett. 2018, 18, 3800-3806.

(35) Girard, A.; Lermé, J.; Gehan, H.; Mermet, A.; Bonnet, C.; Cottancin, E.; Crut, A.; Margueritat, J. Inelastic Light Scattering by Multiple Vibrational Modes in Individual Gold Nanodimers. J. Phys. Chem. C 2019, 123, 14834-14841.

(36) Sönnichsen, C.; Alivisatos, A. P. Gold Nanorods as Novel Nonbleaching Plasmon-Based Orientation Sensors for Polarized Single-Particle Microscopy. Nano Lett. 2005, 5, 301-304.

(37) Schubert, O.; Becker, J.; Carbone, L.; Khalavka, Y.; Provalska, T.; Zins, I.; Sönnichsen, C. Mapping the Polarization Pattern of Plasmon Modes Reveals Nanoparticle Symmetry. Nano Lett. 2008, 8, 2345-2350.

(38) Chang, W.-S.; Ha, J. W.; Slaughter, L. S.; Link, S. Plasmonic Nanorod Absorbers as Orientation Sensors. Proc. Natl. Acad. Sci. 2010, 107, 2781-2786.

(39) Lombardi, A.; Loumaigne, M.; Crut, A.; Maioli, P.; Del Fatti, N.; Vallée, F.; Spuch-Calvar, M.; Burgin, J.; Majimel, J.; Tréguer-Delapierre, M. Surface Plasmon Resonance Properties of Single Elongated Nano-Objects: Gold Nanobipyramids and Nanorods. Langmuir 2012, 28, 9027-9033.

(40) Baida, H.; Billaud, P.; Marhaba, S.; Christofilos, D.; Cottancin, E.; Crut, A.; Lermé, J.; Maioli, P.; Pellarin, M.; Broyer, M.; et al. Quantitative Determination of the Size Dependence of Surface Plasmon Resonance Damping in Single Ag@SiO2 Nanoparticles. Nano Lett. 2009, 9, 3463-3469.

(41) Juvé, V.; Cardinal, M. F.; Lombardi, A.; Crut, A.; Maioli, P.; Pérez-Juste, J.; Liz-Marzán, L. M.; Del Fatti, N.; Vallée, F. Size-Dependent Surface Plasmon Resonance Broadening in Nonspherical Nanoparticles: Single Gold Nanorods. Nano Lett. 2013, 13, 2234-2240.

(42) Yu, K.; Zijlstra, P.; Sader, J. E.; Xu, Q. H.; Orrit, M. Damping of Acoustic Vibrations of Immobilized Single Gold Nanorods in Different Environments. Nano Lett. 2013, 13, 2710-2716.

(43) Major, T. A.; Crut, A.; Gao, B.; Lo, S. S.; Del Fatti, N.; Vallée, F.; Hartland, G. V. Damping of the Acoustic Vibrations of a Suspended Gold Nanowire in Air and Water Environments. Phys. Chem. Chem. Phys. 2013, 15, 4169-4176. 
(44) Medeghini, F.; Crut, A.; Gandolfi, M.; Rossella, F.; Maioli, P.; Vallée, F.; Banfi, F.; Del Fatti, N. Controlling the Quality Factor of a Single Acoustic Nanoresonator by Tuning Its Morphology. Nano Lett. 2018, 18, 5159-5166.

(45) Wang, J.; Yu, K.; Yang, Y.; Hartland, G. V.; Sader, J. E.; Wang, G. P. Strong Vibrational Coupling in Room Temperature Plasmonic Resonators. Nat. Commun. 2019, 10, 1-8.

(46) Yi, C.; Su, M.-N.; Dongare, P. D.; Chakraborty, D.; Cai, Y.-Y.; Marolf, D. M.; Kress, R. N.; Ostovar, B.; Tauzin, L. J.; Wen, F.; et al. Polycrystallinity of Lithographically Fabricated Plasmonic Nanostructures Dominates Their Acoustic Vibrational Damping. Nano Lett. 2018, 18, 34943501.

(47) Davletshin, Y. R.; Lombardi, A.; Cardinal, M. F.; Juvé, V.; Crut, A.; Maioli, P.; Liz-Marzán, L. M.; Vallée, F.; Del Fatti, N.; Kumaradas, J. C. A Quantitative Study of the Environmental Effects on the Optical Response of Gold Nanorods. ACS Nano 2012, 6, 8183-8193.

(48) Hu, W.; Sarveswaran, K.; Lieberman, M.; Bernstein, G. H. Sub-10 nm Electron Beam Lithography Using Cold Development of Poly(Methylmethacrylate). J. Vac. Sci. Technol. B Microelectron. Nanom. Struct. 2004, 22, 1711.

(49) Okoroanyanwu, U. Chemistry and Lithography; Bellingham, Wash. : SPIE Press, 2010.

(50) Muskens, O. L.; Del Fatti, N.; Vallée, F. Femtosecond Response of a Single Metal Nanoparticle. Nano Lett. 2006, 6, 552-556.

(51) Hopkins, B.; Liu, W.; Miroshnichenko, A. E.; Kivshar, Y. S. Optically Isotropic Responses Induced by Discrete Rotational Symmetry of Nanoparticle Clusters. Nanoscale 2013, 5, 6395-6403.

(52) Rahmani, M.; Yoxall, E.; Hopkins, B.; Sonnefraud, Y.; Kivshar, Y.; Hong, M.; Phillips, C.; Maier, S. A.; Miroshnichenko, A. E. Plasmonic Nanoclusters with Rotational Symmetry: PolarizationInvariant Far-Field Response vs Changing near-Field Distribution. ACS Nano 2013, 7, 1113811146

(53) Awada, C.; Popescu, T.; Douillard, L.; Charra, F.; Perron, A.; Yockell-Lelièvre, H.; Baudrion, A. L.; Adam, P. M.; Bachelot, R. Selective Excitation of Plasmon Resonances of Single Au Triangles by Polarization-Dependent Light Excitation. J. Phys. Chem. C 2012, 116, 14591-14598.

(54) Johnson, P. B.; Christy, R. W. Optical Constants of the Noble Metals. Phys. Rev. B 1972, 6, 43704379.

(55) Crut, A.; Maioli, P.; Vallée, F.; Del Fatti, N. Linear and Ultrafast Nonlinear Plasmonics of Single 
Nano-Objects. J. Phys. Condens. Matter 2017, 29, 123002.

(56) Goubet, N.; Tempra, I.; Yang, J.; Soavi, G.; Polli, D.; Cerullo, G.; Pileni, M. P. Size and Nanocrystallinity Controlled Gold Nanocrystals: Synthesis, Electronic and Mechanical Properties. Nanoscale 2015, 7, 3237-3246.

(57) Ahmed, A.; Pelton, M.; Guest, J. R. Understanding How Acoustic Vibrations Modulate the Optical Response of Plasmonic Metal Nanoparticles. ACS Nano 2017, 11, 9360-9369. 


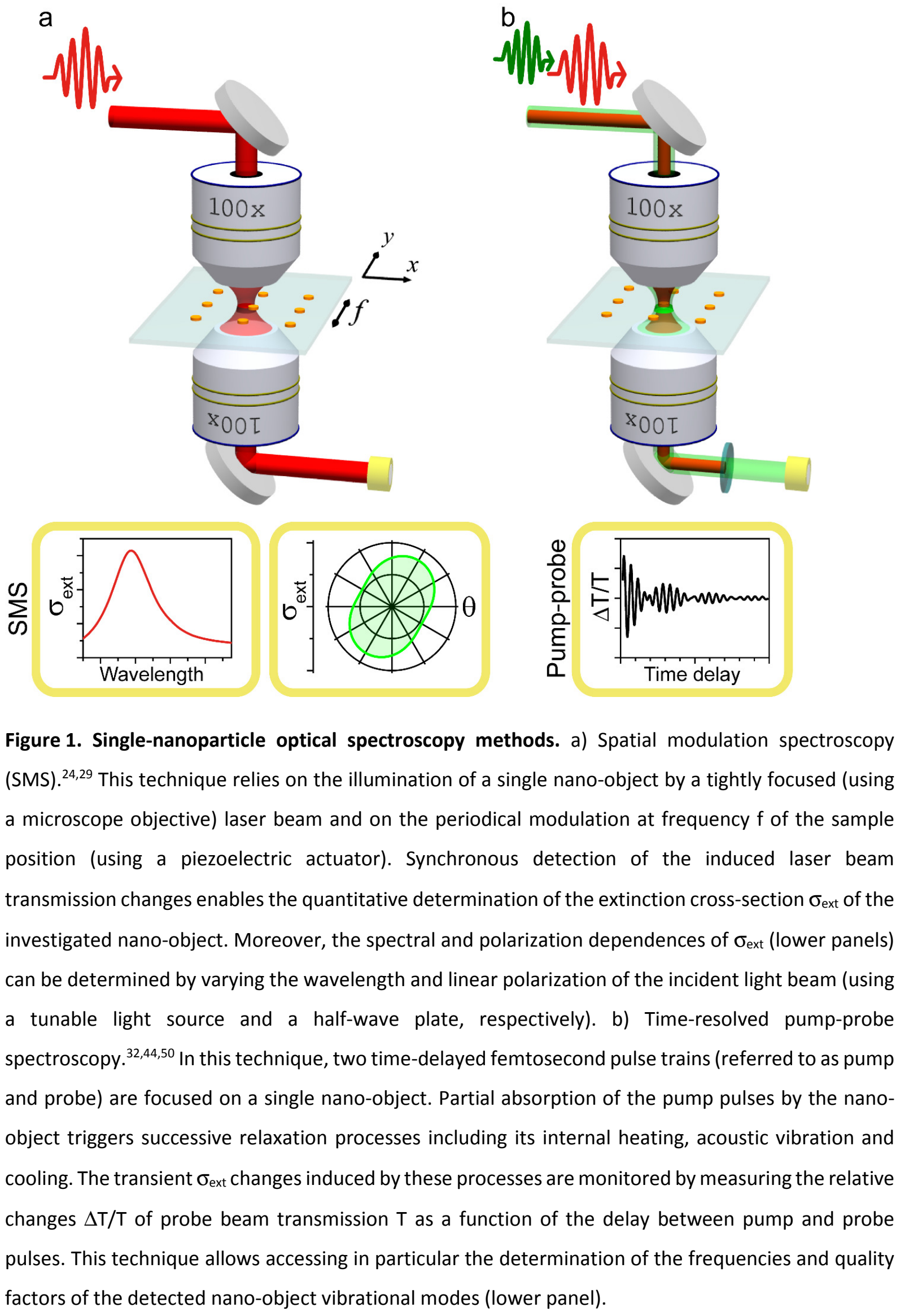




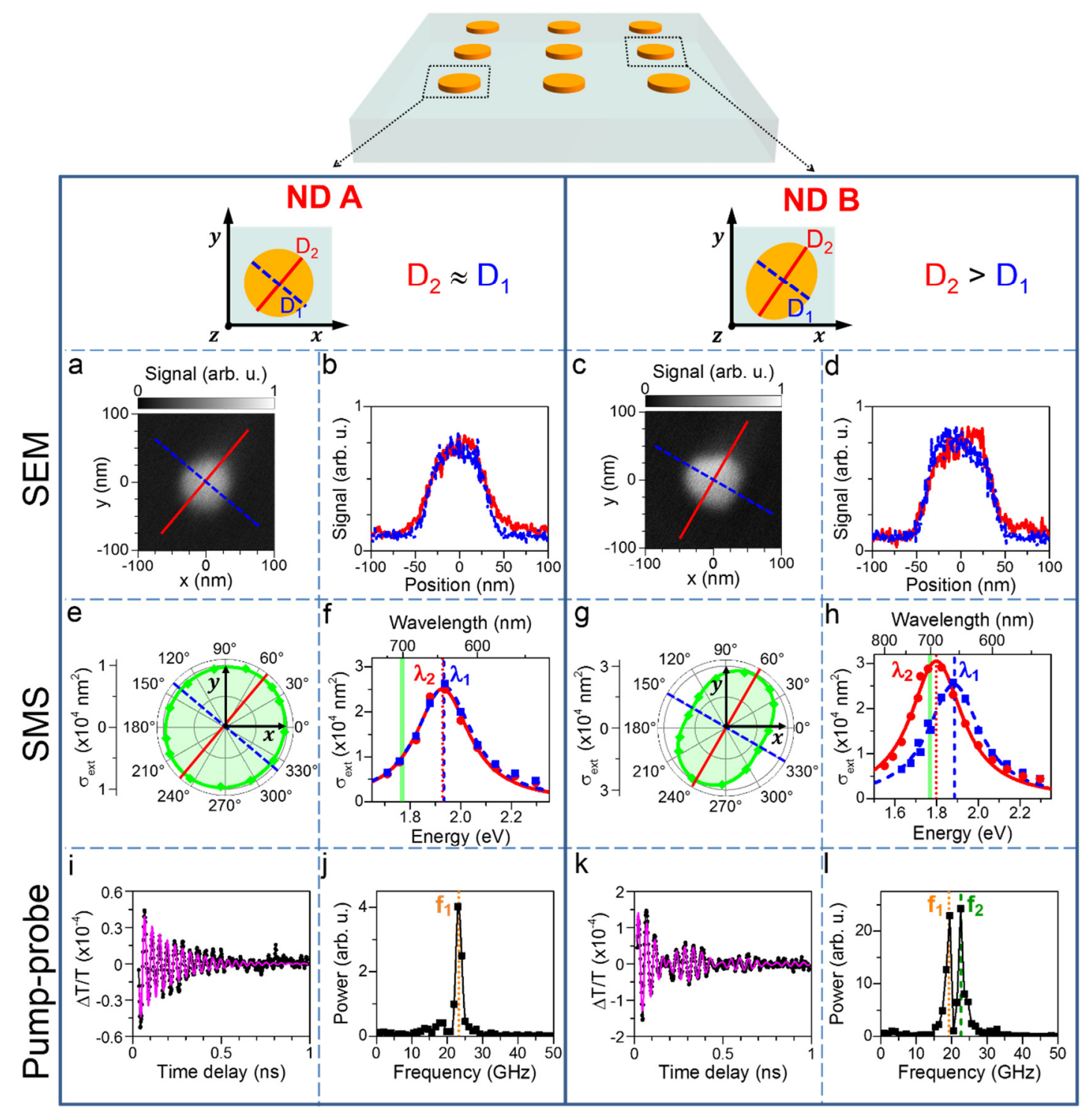

Figure 2. SEM, SMS and pump-probe spectroscopy of two nominally identical gold NDs lithographically defined on a sapphire substrate, with quasi-circular (ND A, left column), and noncircular (ND B, right column) sections. a) SEM image of ND A. b) Intensity profile along directions corresponding to the major (red) and minor (blue) axes of the NDs. c-d) Same for ND B. e) Polarization dependence of ND A extinction at $700 \mathrm{~nm}$ wavelength, measured using $a \approx 10^{-3} \mathrm{~J} / \mathrm{m}^{2}$ incident beam fluence. f) Extinction spectra measured for ND A for the two orthogonal polarization directions corresponding to the major and minor axes of the NDs (same color code as in a-b). Lines correspond to Lorentzian fits in the energy domain g-h) Same for ND B. i) Oscillating part of the time-resolved signal measured for ND A using pump-probe spectroscopy (with $\lambda_{p}=820 \mathrm{~nm}$ pump wavelength, $F_{p} \approx 1$ $\mathrm{J} / \mathrm{m}^{2}$ incident pump fluence and $\lambda_{\mathrm{pr}}=610 \mathrm{~nm}$ probe wavelength, this $\lambda_{\mathrm{pr}}$ choice optimizing the sensitivity of time-resolved signals to ND vibrations). j) Fourier transform. k-I) Same for ND B (same $\lambda_{p}, \lambda_{p r}$ and $F_{p}$ values). 

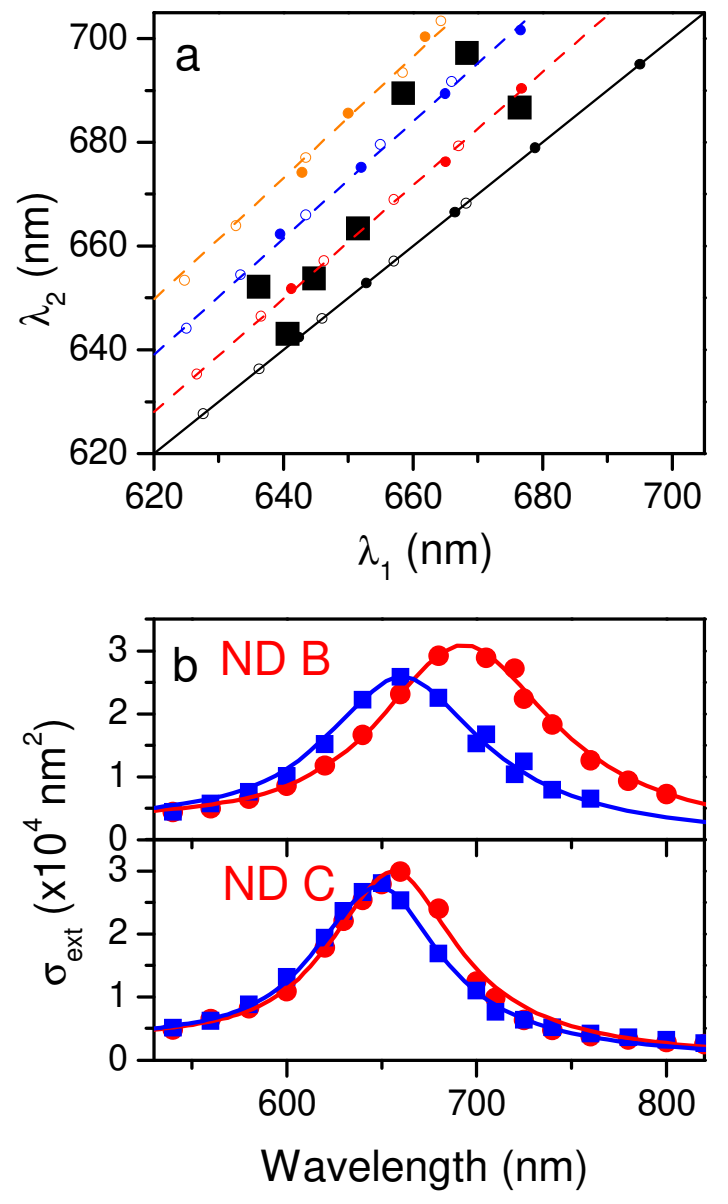

Figure 3. Plasmonic properties of individual gold NDs lithographed on a sapphire substrate. a) Experimental SPR positions $\lambda_{1}$ and $\lambda_{2}$ of seven individual NDs of same nominal dimensions $D=70 \mathrm{~nm}$ and $\mathrm{h}=20 \mathrm{~nm}$ (black squares, the symbol size corresponding to experimental uncertainties), measured using SMS for the two orthogonal incident light polarizations yielding maximal and minimal extinction at $700 \mathrm{~nm}$ wavelength. Also shown are the computed SPR positions using optical FEM simulations on $\mathrm{h}=20 \mathrm{~nm}$ (full circles) and $\mathrm{h}=25 \mathrm{~nm}$ (hollow circles) NDs with circular or elliptical section of different $D_{1}$ and $D_{2}$ values, such that $D_{2} / D_{1}=1$ (black circles, corresponding to the case of circular disk with degenerate SPRs, i.e. $\lambda_{1}=\lambda_{2}$ ), 1.05 (red circles), 1.1 (blue circles) and 1.15 (orange circles). Lines correspond to linear fits of the simulated results. b) Extinction spectra of two individual gold NDs (top: ND B, bottom: ND C), measured for two orthogonal light polarizations yielding maximal (red circles) and minimal (blue squares) extinction at $700 \mathrm{~nm}$ wavelength, and FEM-simulated spectra (red and blue lines) obtained using morphological parameters yielding an optimal reproduction of the quantitative experimental spectra: $h=21.3 \mathrm{~nm}, D_{1}=79.9 \mathrm{~nm}$ and $D_{2} / D_{1}=1.14(N D B)$ and $h=23.7 \mathrm{~nm}, D_{1}=78.3 \mathrm{~nm}$ and $D_{2} / D_{1}=1.04$ (ND C). 


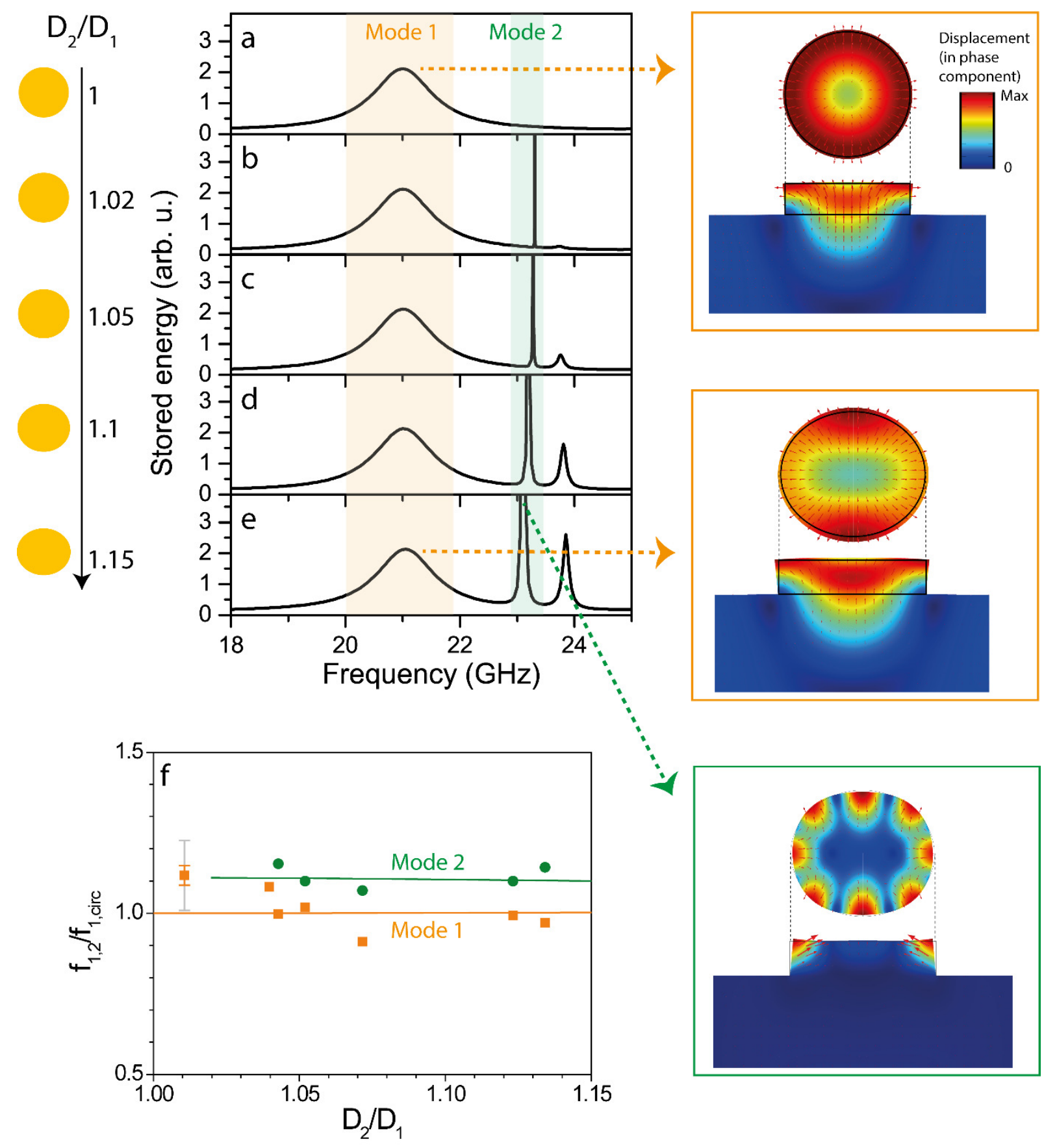

Figure 4. Vibrational response of individual gold NDs lithographed on a sapphire substrate. (a-e) FEM-computed ND vibrational spectra for $h=20 \mathrm{~nm}, D_{\mathrm{eq}}=80 \mathrm{~nm}$ and $D_{2} / D_{1}=1$ (a), 1.02 (b), 1.05 (c), 1.1 (d) and 1.15 (e). Only the $18-25 \mathrm{GHz}$ frequency range relevant for the analysis of pump-probe experiments is shown. For circular NDs, a single mode (mode 1 ) is predicted to be excited in this frequency range (a), with a displacement profile (right) symmetrical about the ND axis (top slice, showing displacement in the ND top surface). Three modes appear in the simulated spectra of NDs with elliptical sections. The lowest frequency $(\approx 21 \mathrm{GHz})$ one corresponds to mode 1 . The intermediate frequency one $(\approx 23 \mathrm{GHz})$ is referred to as mode 2 . The highest-frequency mode (mode 3 at $\approx 24 \mathrm{GHz}$ ) has an exiguous spectral area as compared to the two previous modes. f) Measured and FEM- 
computed frequencies of modes 1 ( $f_{1}$, orange) and 2 ( $f_{2}$, green). The frequencies measured for the two experimentally detected vibrational modes of individual Au NDs on sapphire are shown as orange squares (mode 1 ) and green circles (mode 2, not detected for the two NDs with the lowest $D_{2} / D_{1}$ values). FEM-computed $f_{1}$ and $f_{2}$ values, deduced from the vibrational spectra shown in panels a-e, are shown as orange and green lines, respectively. All frequencies were normalized by division by the frequency $f_{1, \text { circ }}$ of the vibrational mode of a circular ND of height $h$ and equivalent diameter $D_{e q}=\left(D_{1} D_{2}\right)^{1 / 2}$ (using for experimental data the $h, D_{1}$ and $D_{2}$ values deduced from the analysis of measured extinction spectra, Fig. 3b). Grey errors bars (shown only on one data point for simplicity) include uncertainties originating from both acoustic frequency $f_{1,2}$ experimental determination (shown in orange) and $\mathrm{f}_{1, \text { circ }}$ computation, based on ND sizes estimated from optical measurements, assuming a cylindrical ND shape. 
TOC

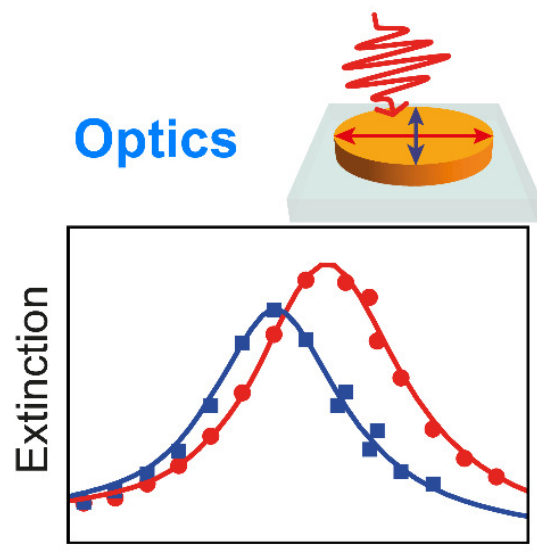

Wavelength
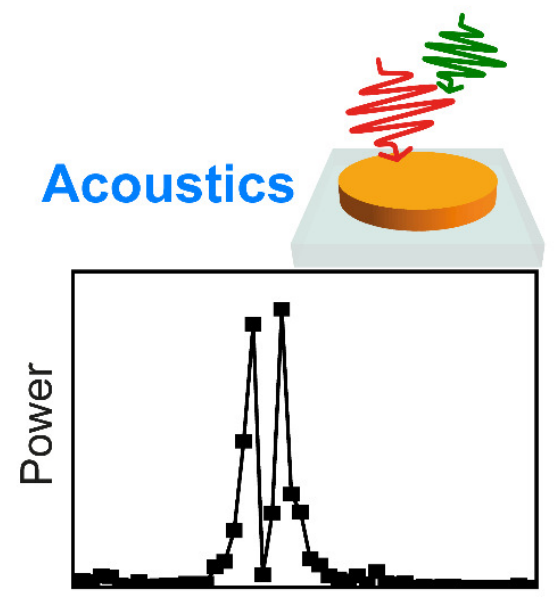

Frequency 